\title{
IDENTIFICATION OF LÜDERS BANDS USING DIGITAL IMAGE CORRELATION
}

\author{
Tin Brlić*, Dimitri Debruyne ${ }^{* *}$, Pascal Lava ${ }^{* * *}$, Stoja Rešković*, Ivan Jandrlić* \\ * University of Zagreb, Faculty of Metallurgy, Sisak, Croatia \\ ${ }^{* *}$ KU Leuven, Ghent, Belgium \\ ${ }^{* * *}$ MatchID NV, Ghent, Belgium \\ corresponding author: Tin Brlić, e-mail: tbrlic@simet.hr
}

\begin{abstract}
This paper presents the characterization of Lüders bands by digital image correlation on niobium microalloyed steel during a static tensile testing. Digital image correlation with the qualitative and quantitative analysis of the Lüders bands on the microalloyed steel was proved as a very precise and suitable method for determining the strain amount in the deformation zone. In this research was determined that the strain amount is the highest in the area behind the Lüders band front and the lowest in the area in front of the Lüders band.
\end{abstract}

Keywords: Lüders bands, digital image correlation, microalloyed steel

\section{INTRODUCTION}

In metalworking applications, an inhomogeneous plastic deformation, i.e. Lüders bands, can pose a significant problem. Different materials, such as low carbon steels [1], aluminium alloys [2], transformation induced plasticity (TRIP) steels [3], shape memory alloys [4] and copper [5], can show the appearance of the Lüders bands during plastic deformation. This phenomenon can be attributed to the localisation of deformation and pinning and unpinning interactions between dislocations and solutes [6].

Recently, various methods have been used to determine the Lüders bands of which the most commonly used are thermography [7] and digital image correlation [8]. Digital Image Correlation (DIC) is a non-contact, easy to use and cost-effective optical technique for measuring strains and displacements. It is used, amongst others, for the characterization of material properties and the monitoring of components and structures. Under the right experimental conditions, DIC can provide accurate and precise strain measurements in both indoor and outdoor environments [9]. The method offers opportunities for exploring complex full-field displacements and deformations at the surface of an object under both 2D and 3D loading conditions, depending on the number of cameras used. The captured images are computationally processed in the 
correlation software and results are typically represented in the form of full-field displacements and strain fields at the surface. DIC has a lot of advantages over traditional strain measurements and is an increasingly used tool in the engineering community with a widespread application such as mechanical engineering, material science, material identification, biomedical and biomechanical sciences [10].

DIC has been used before to identify and characterize the Lüders bands. The characteristics of the Lüders bands, e.g. the Lüders strain, the morphology of the Lüders bands, the Lüders bands velocity, band growth mechanisms, Lüders band formation and propagation can theoretically be determined with the aid of DIC. Cai et al. [11] have studied the thickness dependence of the Lüders effect in the Al-based alloy. The Lüders band growth mechanism, in the effective width and active zone, has also been successfully characterized in [8]. Srinivasan et al. [12] have studied the Lüders band formation on IS 2062 grade-E250 B steel at the start and just before the plastic deformation.

The aim of this paper is to demonstrate the feasibility of using DIC for the characterization and identification of Lüders bands during the static tensile testing. In this paper, the experimental data and the results of analysis on the deformation zone with Lüders band of niobium microalloyed steel are provided.

\section{EXPERIMENTAL}

The experiments were performed on the static tensile machine EU 40mod with a nominal force value of $400 \mathrm{kN}$. For the DIC measurements, the digital camera with CMOS sensor (3.2 Mpixel resolution) was used together with the MatchID software for processing. The static tensile testing was performed on the niobium microalloyed flat test pieces with rectangular cross section samples cut off from the hot rolled steel strip with the original gauge length of $45 \mathrm{~mm}$, original width of $20 \mathrm{~mm}$ and thickness of 3 $\mathrm{mm}$. Crosshead speed at room temperature was $20 \mathrm{~mm} / \mathrm{min}$. The static tensile test was performed in accordance with EN ISO 68921:2009.

As the measurements were done with one camera only, great care was taken to align the camera perpendicular to the specimen. The samples were prepared by coating the black matte coating on the clear surface of total length to avoid possible reflection. The white speckles were applied on the surface of total length after the black matte coating to achieve the best contrast. The dry undercoat was of great importance allowed avoiding speckle layer diffuse with the undercoat which can result in the blurred image.

\section{RESULTS AND DISCUSSION}

First of all it is necessary to choose the reference and the deformed images obtained with the digital camera. On the reference image, prior to the digital image correlation process, it is necessary to define a region of interest where Lüders bands are expected to appear for the accurate and precise bands characterization, Figure 1. For the successful processing and correlating of the images, the appropriate parameters such as noise evaluation, subset and step size, correlation options and strain calculation are required.

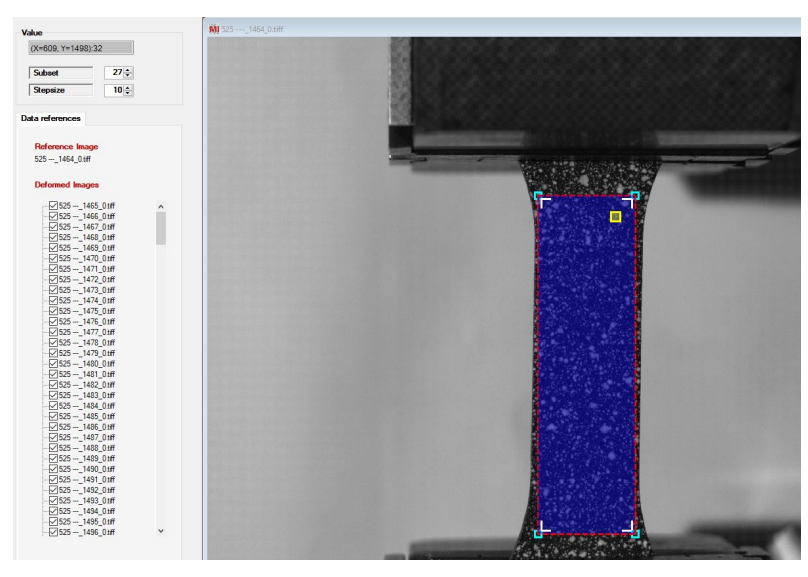

Figure 1. Defining the region of interest in software MatchID 
By setting parameters and marking images, the digital image correlation process started with MatchID software where Lüders band was detected in niobium microalloyed steel. Figure 2 represents the qualitative analysis of the Lüders band formation and the propagation from the upper end of the sample to the lower end of the sample. The amount of the Lüders strain can be determined at any time of deformation in the region of interest by the method of digital image correlation.

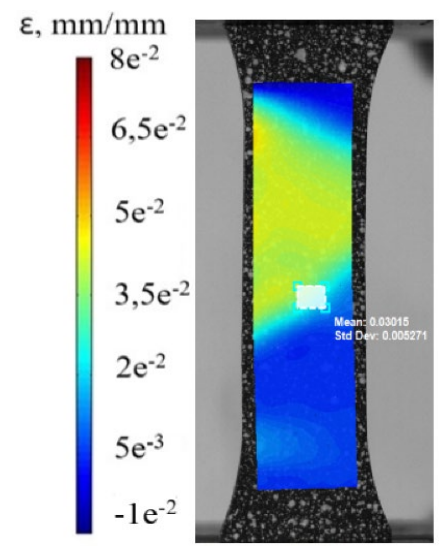

Figure 2. Qualitative analysis of the Lüders band

In addition to qualitative analysis, the quantitative analysis of the Lüders band can be determined with the software MatchID, as presented in Figure 3. At the start of the deformation, no significant change in the amount of strain is seen, until a sudden jump appears, indicating the onset of the Lüders band propagation through the part marked in Figure 2.

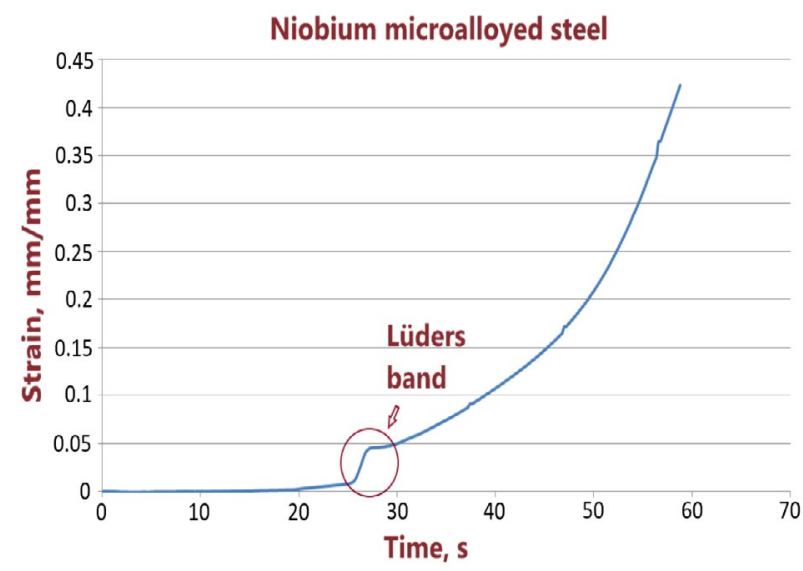

Figure 3. Quantitative analysis of the Lüders band
Lüders bands can be characterized in different regions. It is possible to determine the strain amount at the Lüders front, in front of the Lüders front (undeformed area) and behind the Lüders front (deformed area), Figure 4. The Lüders band gradually propagates along the sample from the upper end of the sample to the lower end of the sample. It is clearly visible that the Lüders band propagates first in the area behind the Lüders band front (blue mark and line). By further propagation, the Lüders band reaches the green mark point (green line). After that, in the purple area, where the increase of strain amount occurs later in relation to the blue and green mark clearly reveals that the Lüders band was propagated later in this area. The inset in Figure 4 shows that at the same time (orange triangles and circle) the strain amount is the highest in the blue area and the lowest in the purple area because the Lüders band first propagates through the blue area and after that through the green and purple area. As the Lüders band propagates through the blue area a certain deformation is achieved in this area. The purple area represents the elastic area (undeformed region) and there is no significant change in the strain amount.

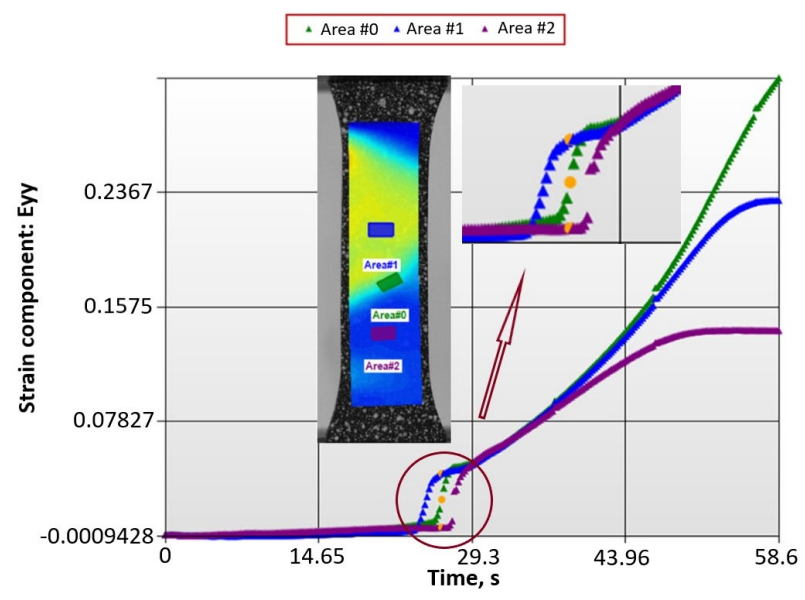

Figure 4. Strain determination behind, in front of and at the Lüders band front

\section{CONCLUSION}

Digital image correlation was shown to be a very suitable method for the determination and 
characterization of Lüders bands using MatchID software package. The strain amount is the highest in the area behind the Lüders band front and the lowest in the elastic area where no strain increase was observed. Research has shown that in the area in front of the Lüders band front is still the area of elastic strain. In the area behind the Lüders band front elasto-plastic strain is occurred. Due to the appearance of plastic strain on the Lüders band front and behind the Lüders band, strains are greater than in the elastic area. Strain amount does not change significantly behind the Lüders band front from Lüders band formation until the end of the Lüders band propagation through the deformation zone.

\section{REFERENCES}

[1] N. Tsuchida, Y. Tomota, K. Nagai, K. Fukaura, A simple relationship between Lüders elongation and work-hardening rate at lower yield stress, Scripta Materialia 54(2006) 1, 57-60.

[2] J. Coër, P.Y. Manach, H. Laurent, M.C. Oliveira, L.F. Menezes, Piobert-Lüders plateau and Portevin-Le Chatelier effect in an Al-Mg alloy in simple shear, Mechanics Research Communications 48(2013), 1-7.

[3] E. Emadoddin, A. Akbarzadeh, G.H. Daneshi, Correlation between Luder strain and retained austenite in TRIPassisted cold rolled steel sheets, Materials Science and Engineering A 447(2007) 1-2, 174-179.

[4] L. Zheng, Y. He, Z. Moumni, Luderslike band front motion and fatigue life of pseudoelastic polycrystalline NiTi shape memory alloy, Scripta Materialia 123(2016), 46-50.

[5] Y.Z. Tian, S. Gao, L.J. Zhao, S. Lu, R. Pippan, Z.F. Zhang, N. Tsuji, Remarkable transitions of yield behavior and Lüders deformation in pure $\mathrm{Cu}$ by changing grain sizes, Scripta Materialia 142(2018), 88-91.

[6] T. Cheng, X. Xu, Y. Cai, S. Fu, Y. Gao, Y. Su, Y. Zhang, Q. Zhang,
Investigation of Portevin-Le Chatelier effect in 5456 Al-based alloy using digital image correlation, Optics and Lasers in Engineering 65(2015), 89-92.

[7] J. Petit, D. Wagner, N. Ranc, G. Montay, M. François, Comparison of different techniques for the monitoring of the Lüders bands development, Proceedings book of the $13^{\text {th }}$ International Congress on Fracture 2013, IFC-13, Chinese Society of Theoretical and Applied Mechanics, Beijing, 16 - 21 June 2013, 3797-3809.

[8] N. Srinivasan, R. Narayanaswamy, B. Venkatraman, Study on local zones constituting to band growth associated with inhomogeneous plastic deformation, Materials Letters 105(2013), 209-212.

[9] N. McCormick, J. Lord, Digital Image Correlation, Materials Today 13(2010) $12,52-54$.

[10] MatchID, Theory manual, Accessible on Internet:

http://matchidmbc.be/Documentation/do ku.php?id=matchid:training:lessons:dic, Accessed: March 2,' 2018.

[11] Y.-L. Cai, S.-L. Yang, S.-H. Fu, Q.-C. Zhang, The Influence of Specimen Thickness on the Lüders effect of a 5456 Al-based alloy: Experimental Observations, Metals 6(2016) 5, 1-12.

[12] N. Srinivasan, R. Narayanaswamy, B. Venkatraman, Advanced imaging for early prediction and characterization of zone of Lüders band nucleation associated with pre-yield microstrain, Materials Science and Engineering A 561(2013), 203-211.

\section{Acknowledgements}

This work has been fully supported by Croatian Science Foundation under the project IP-2016-06-1270. 